\title{
Energy Efficient Direction-Based Topology Control Algorithm for WSN
}

\author{
Muhammad Asghar Khan1, Sadam Hussain² \\ ${ }^{1}$ IECS, University of Science \& Technology Bannu, Bannu, Pakistan \\ ${ }^{2}$ Department of Computer Science, Islamia College University Peshawar, Peshawar, Pakistan \\ Email: asghar_surani@yahoo.com, imislamian@gmail.com
}

How to cite this paper: Khan, M.A. and Hussain, S. (2020) Energy Efficient Direction-Based Topology Control Algorithm for WSN. Wireless Sensor Network, 12, 37-47. https://doi.org/10.4236/wsn.2020.123003

Received: March 4, 2020

Accepted: March 24, 2020

Published: March 27, 2020

Copyright () 2020 by author(s) and Scientific Research Publishing Inc. This work is licensed under the Creative Commons Attribution International License (CC BY 4.0).

http://creativecommons.org/licenses/by/4.0/

\begin{abstract}
A wireless sensor network consists of hundreds or thousands of small nodes which could either have a static or dynamic position. These nodes are deployed through normal or random distribution to report events of a particular area to the base station through sink nodes. Having limited onboard energy of sensor nodes, conservation of energy in wireless sensor network is necessary. For this purpose, a new algorithm is proposed titled Energy-EfficientDirection-Based-Topology-Control-Algorithm (EEDBTC). In proposed algorithm, direction is the main concern whenever an event occurs the node will send data in the direction of base station so that less energy is consumed. The results of the same were compared with customary dense wireless sensor network, color based WSNs and it was observed that this algorithm is much better than previous topology control algorithms used.
\end{abstract}

\section{Keywords}

Sensor Nodes, Topology Control, Energy Conservation, Energy Efficiency

\section{Introduction}

Sensors are tiny devices, responsible for substantial happenings or occurrences like humidity, temperature, vibrations, pressure etc. and then produce a signal that can be monitored, calculated or transformed to a new type of signal [1]. A wireless sensor network is a collection of sensor nodes deployed over a huge environmental area, called Sensor Field. A sensor Network composed of three main components, classified as: 1) a sensing subsystem, 2) a processing subsystem and 3) a communication subsystem [2]. In general, a sensing subsystem is used for physical data gaining from the desired environment. Processing unit is responsible for data processing and storage. Wireless communication subsystem is used 
for data transmission purposes. To perform all these tasks energy is required which is supplied by the power supply unit. In sensor node, a small battery is used, although it has to operate the all the mentioned systems but it has energy restraint [3]. Once the nodes are deployed in area under observation then it is impossible to recharge the battery. In some application, the sensor network required long lifetime to complete the targeted mission. For this the network lifetime should be enlarged. Therefore, to the design the wireless sensor networks the energy conservation is an important problem to be addressed. From experiments it is bring into our knowledge that energy need for processing thousands operations in a sensor node is about equal as for transmitting information of a single bit [4]. Consumption of energy in the sensing subsystem depends on the type of sensor use. As for as energy is concern, the consumption of energy in processing is high as compare to sensing subsystem so it can be neglected. Mainly it relays on the communication subsystems. Energy needed for data transmission is lower than that of energy needed for sensing. Hence it is concluded that all the techniques used for energy conservation mostly pay attention on the communication subsystem and the sensing subsystem. WSN is consisting of a large number of nodes and a sink node also called base station. Sensor nodes can be deployed in an area under observation, termed as sensor field as shown in Figure 1. Data is transferred from sensor nodes to base station using Multi-hop communication system [5] [6].

The following five components can be found mostly in every sensor node [7] as shown in the Figure 2.

- Memory.

- Controller.

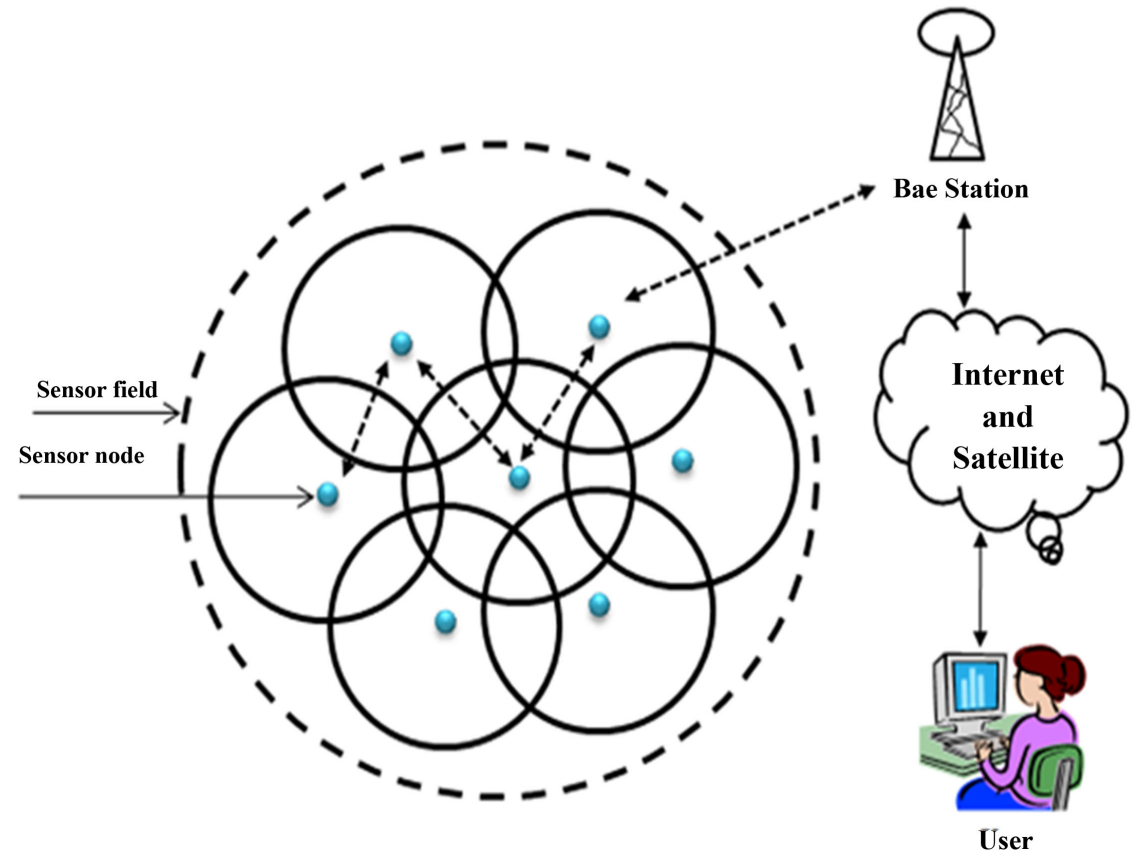

Figure 1. Typical wireless sensor network. 


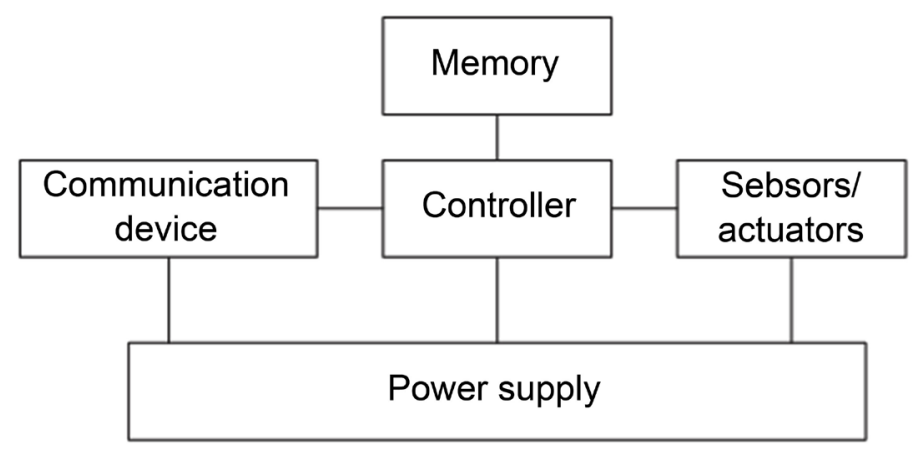

Figure 2. Components of sensor node.

- Power supply.

- Communication device.

- Sensing device.

\section{Literature Review}

In [8] the author gave different procedures for developing topologies, with minimized link interference, either connected or a spanner for Euclidean length to find the connected spanning sub graph of the interference graph. To estimate the Euclidean minimum spanning tree [9] [10] the author has suggested several new configurations, based on $\mathrm{O}(\mathrm{n})$ messages utilizing only local data. To minimize or partially minimize the interference of node or link the author $\mathrm{K}$. Moaveni-Nejad give et al. [11] has defined many algorithms to build a network topology for ad hoc wireless network. The most important goal in topology control is to reduce the interference. Nearly all these related works, however, reflects this issue indirectly: Little interference is often called to be a significance of scarcity or low degree of the made topologies. While Rickenbac et al. [12] explained the direct descriptions of interference. (Both from a sender-centric and a receiver-centric perspective) to check the properties of algorithms and its complexity several models of interference were suggested. The interference model proposed in [13] is built on current network traffic, however, highly depends on the chosen application. The detail of topology control is described in [14]. Generally disk model is used in numerous research techniques [15] [16]. To represent a WSN Unit disk graph model is used. For a true sensor network static communication or sensing range is not applicable.

Nodes in WSNs have limited power that's why its communication and coverage range is lesser than other mobile devices [17]. A sensor node will work as for as its energy remains otherwise it will be of no use if it loses all of its energy. Similarly if the number of such type of nodes increases it will leads to harm the structure of WSN [18]. Due to the resource constraint, conservation of energy in sensor nodes is one of the tricky features of these networks. In this regard several methods and protocols are developed to overcome this important challenge. In power limited network research the main achievement is of minimum energy consumption [19]. WSN is the collection of nodes, deployed closely to each oth- 
er. Every node is surrounded by neighboring nodes. All the nodes communicating directly with their neighbor nodes and as a result a lot of energy is consumed. Due to the close deployment of sensor nodes, various issues may be occurred in the networks i.e. energy consumption, energy holes, unnecessary transmission of data packets [20]. The major issue in barrier coverage is the probability decreasing of undetected overload, limited sensing range, random deployment and insufficient sensor nodes to cover the target area are the three main factors that produce coverage problems in WSNs [21]. To restrict the neighboring nodes different techniques have been used i.e. regulating transmission power, turn off some nodes for a particular time [20]. Nor Azlina et al., discussed various techniques to save and minimize the use of built-in battery power [22].

The deployments of homogeneous WSNs are easy as compared to heterogeneous WSNs. Before real deployment and considering the cost of wireless sensor nodes, the deployment simulation is necessary therefore, to get the best configuration results several configurations should be tested [23].

\section{Proposed Topology Control for WSNs}

According to the new topology control algorithm, direction is the main criterion, data can be send from a node to another node in the direction of Base Station. For this purpose Energy Efficient Direction Based Topology Control (EEDBTC) is developed, this topology control algorithm uses the concept of four quadrants. The coverage area, $2 \pi$ of a node is divided into four quadrants $(\pi / 2$ each) i.e., Quadrant I, II, III \& IV as shown in Figure 3 \& Figure 4. For transmission of data from one node to another or any other sink node, base station will be searched out first and data will be transmitted in the direction of base station i.e. select the quadrant where Base Station (BS) is located. After selecting the desired quadrant the following method will be adopted. Divide the quadrant into three parts i.e. $(\pi / 2) / 3$ means $\pi / 6$. Among these three portion, select the most suitable $\pi / 6$ as shown in Figure 5 \& Figure 6. (Assumption: Assume that all nodes are deployed in such a way that every portion of $\pi / 6$ contains at least
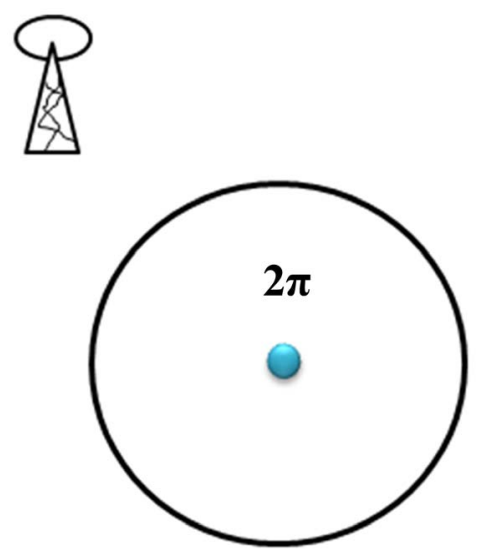

Figure 3. Coverage area $=2 \pi$ 


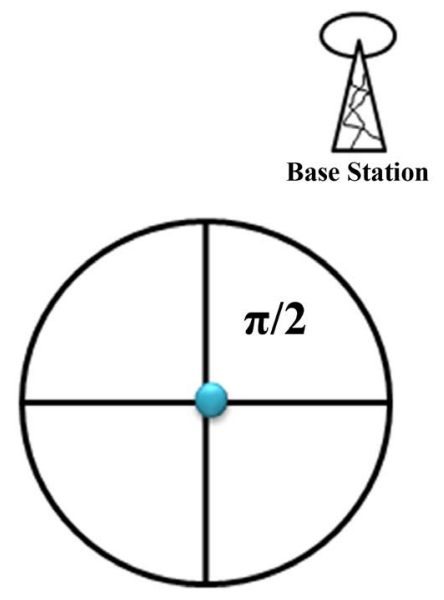

Figure 4. Quadrant $=\pi / 2$.

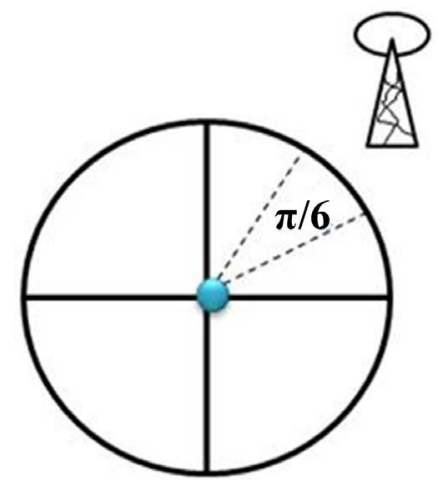

Figure 5. Divide the quadrant into three parts i.e. $(\pi / 2) / 3$ or $\pi / 6$.

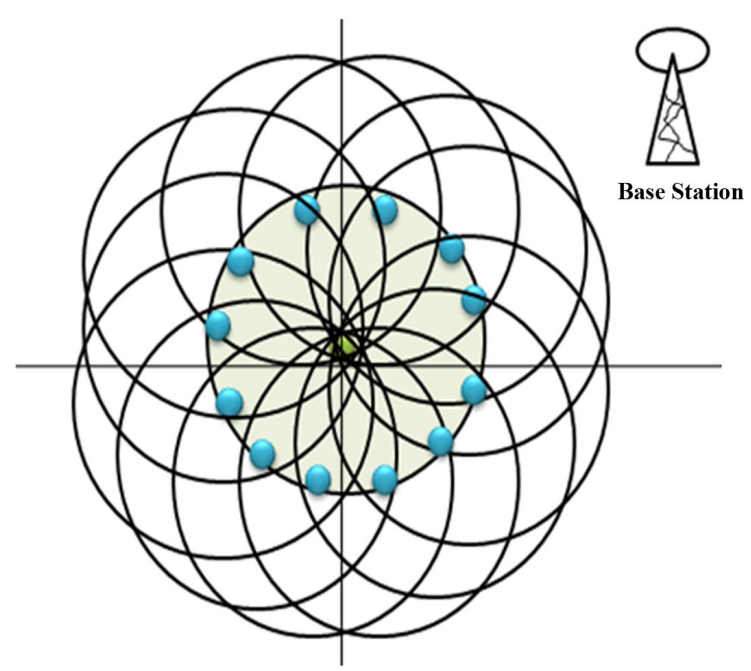

Figure 6. Every portion of $\pi / 6$ contains at least one node.

one node). Send Beacon message to nodes of that region and after receiving ACK from any of them start transmission with it. If all the nodes in the selected $\pi / 6$ are not working due to some reasons then increase the area from $\pi / 6$ to $\pi / 3$ and then to whole quadrant i.e. $\pi / 2$. 


\subsection{Pseudo code of Energy Efficient Direction-Based Topology Control Algorithm}

Suppose that event occurs at the location of node " $\mathrm{X}$ " and the Base station (BS) is located in the fourth Quadrant so that node will send the data in this quadrant only.

$>$ Start

Event occurs at Node $\mathrm{X}$ and wants to send it to the node $\mathrm{Y}$

Divide the coverage area of node $\mathrm{X}$ into four $\mathrm{Qs}$ where $\mathrm{Q}$ is the quadrant

Check the direction of BS w.r.t four Qs

Select the desired Q

Divide the $\mathrm{Q}$ into three equal parts i.e. $(\pi / 2) / 3$

$>$ Search the $\pi / 6$ to check the status of node Y.

If

Available start communication

Else

Select other part i.e. $\pi / 3, \pi / 2$

End

\subsection{Flow Chart of the Proposed Algorithm}

The complete flow of the EEDBTC algorithm is shown at Figure 7.

\section{Results \& Discussion}

The algorithm is evaluated on ten different locations, selected randomly with respect to energy consumed, time taken, and number of packets sent per unit time. The evaluation is done in Visual Studio 12.0, $(\mathrm{C}++)$, using core-i7 processor and $8 \mathrm{~GB}$ of RAM, 2.5GH processing speed of the computer.

\subsection{Evaluation of EEDBTC Algorithm w.r.t. Energy}

During evaluation it was calculated that energy consumed by the following different topologies given in the Table 1 i.e. T-WSN is $33.74 \%$, CBTC-N $27.18 \%$, CBTC-R $29.16 \%$ and EEDBTC is $7.36 \%$. In the bellow table 10, 50, 75, 100, 200, $1000,1500,3000,4000$ are the location where event occurred and bellow the location is energy consumed in joule (Figure 8).

\subsection{Evaluation EEDBTC Algorithm w.r.t. Number of Packet Sent}

The number of packets sent after the event occurs by a node in different topology control as shown in the Table 2 i.e. T-WSN is $62.71 \%$, CBTC-N $12.18 \%$, CBTC-R $16.24 \%$ and EEDBTC is $10.99 \%$. In the bellow table 10, 50, 75, 100, 200, $1000,1500,3000,4000$ are the location where event occurred and bellow the location is number packets sent from location to base stations (Figure 9).

\subsection{Evaluation EEDBTC Algorithm w.r.t. Time (CPU Ticks)}

The number of CPU ticks (time) taken by the mentioned algorithm is shown in 


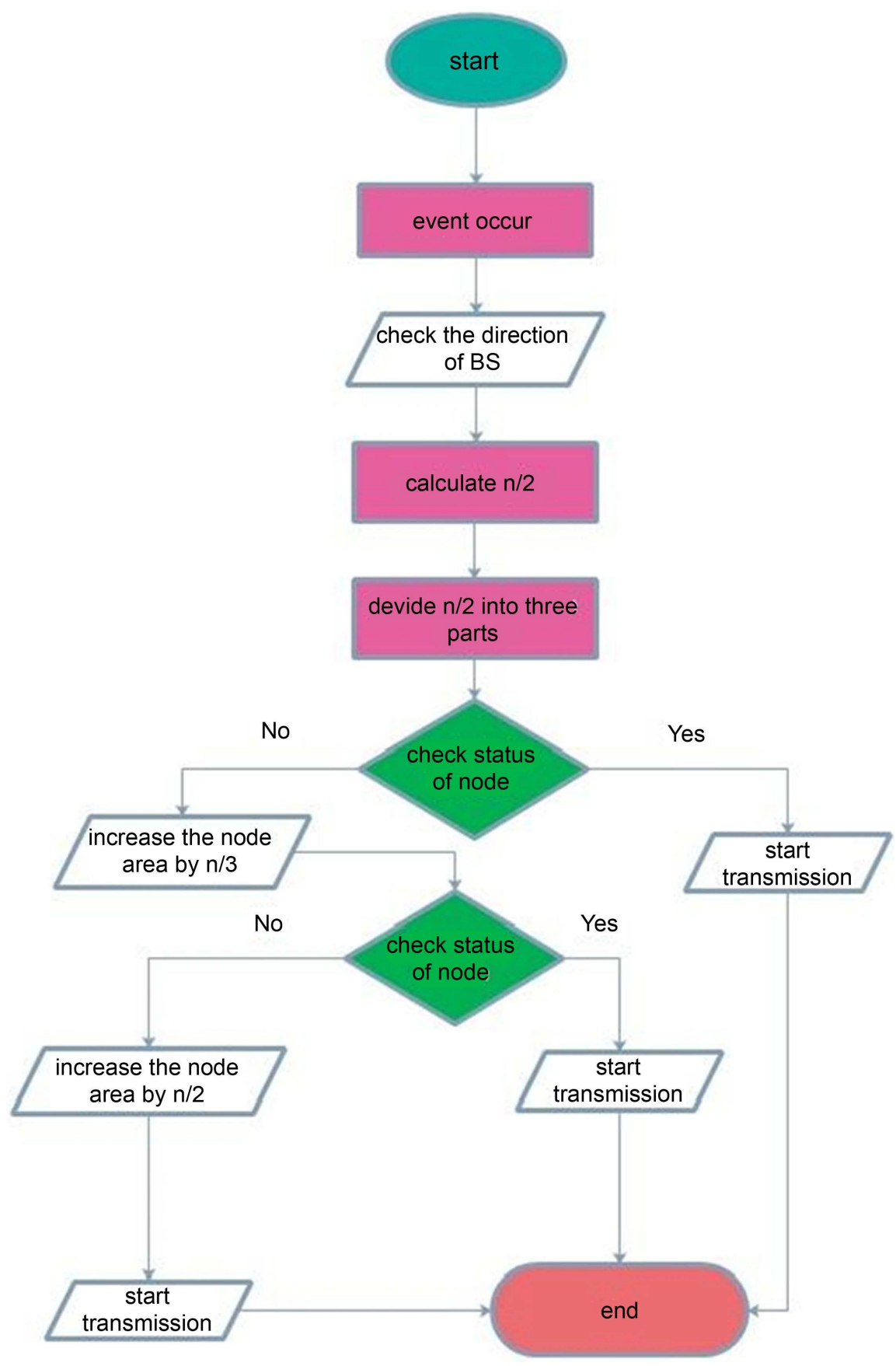

Figure 7. Flow chart diagram of proposed algorithm.

Table 1. Evaluation of EEDBTC algorithm w.r.t. energy.

\begin{tabular}{ccccccccccc}
\hline & \multicolumn{1}{c}{ Location of the nodes where event occur and consumption of energy } \\
\hline Network & 10 & $\mathbf{5 0}$ & $\mathbf{7 5}$ & $\mathbf{1 0 0}$ & $\mathbf{2 0 0}$ & $\mathbf{2 5 0}$ & $\mathbf{1 0 0 0}$ & $\mathbf{1 5 0 0}$ & $\mathbf{3 0 0 0}$ & $\mathbf{4 0 0 0}$ \\
\hline T-WSN & 249 & 245 & 242 & 240 & 230 & 225 & 150 & 100 & 86 & 150 \\
CBTC-N & 149 & 148 & 148 & 147 & 145 & 144 & 129 & 119 & 110 & 120 \\
CBTC-R & 168 & 167 & 166 & 139 & 155 & 159 & 132 & 119 & 113 & 140 \\
EEDBTC & 50 & 47 & 46 & 39 & 43 & 46 & 41 & 17 & 9 & 30 \\
\hline
\end{tabular}


Table 2. Evaluation of EEDBTC algorithm w.r.t. packets.

\begin{tabular}{lccccccccccc}
\hline & \multicolumn{1}{c}{ Location of the node and number packets sent from the location to base station } \\
\hline Network & 10 & 50 & 75 & 100 & 200 & 250 & 1000 & 1500 & 3000 & 4000 \\
\hline T-WSN & 2490 & 2450 & 2425 & 2400 & 2300 & 2250 & 1500 & 1000 & 500 & 1500 \\
CBTC-N & 497 & 489 & 484 & 479 & 459 & 449 & 299 & 199 & 100 & 200 \\
CBTC-R & 680 & 671 & 663 & 399 & 551 & 599 & 328 & 999 & 133 & 401 \\
EEDBTC & 507 & 479 & 469 & 399 & 438 & 461 & 421 & 177 & 99 & 307 \\
\hline
\end{tabular}

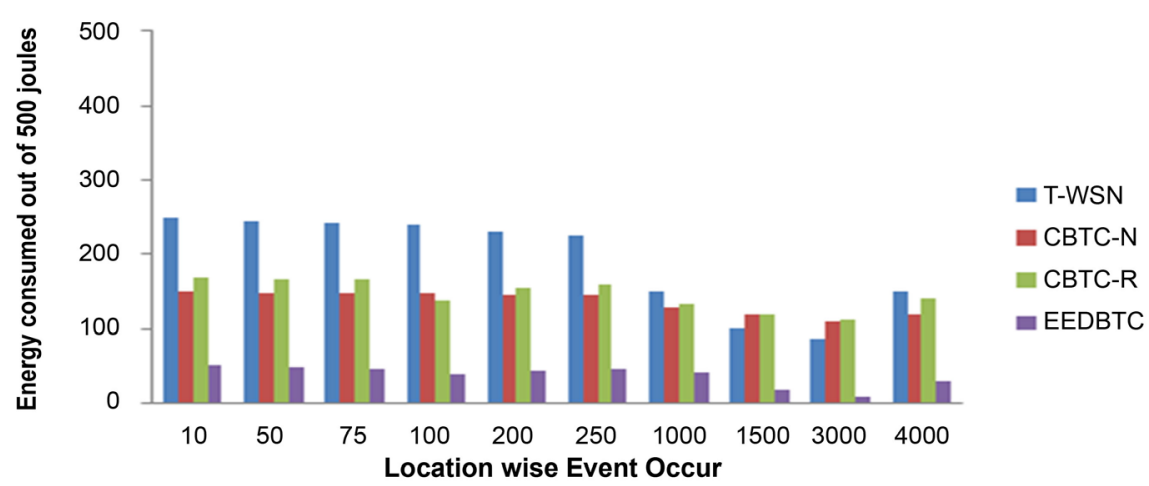

Figure 8. Comparison w.r.t. energy.

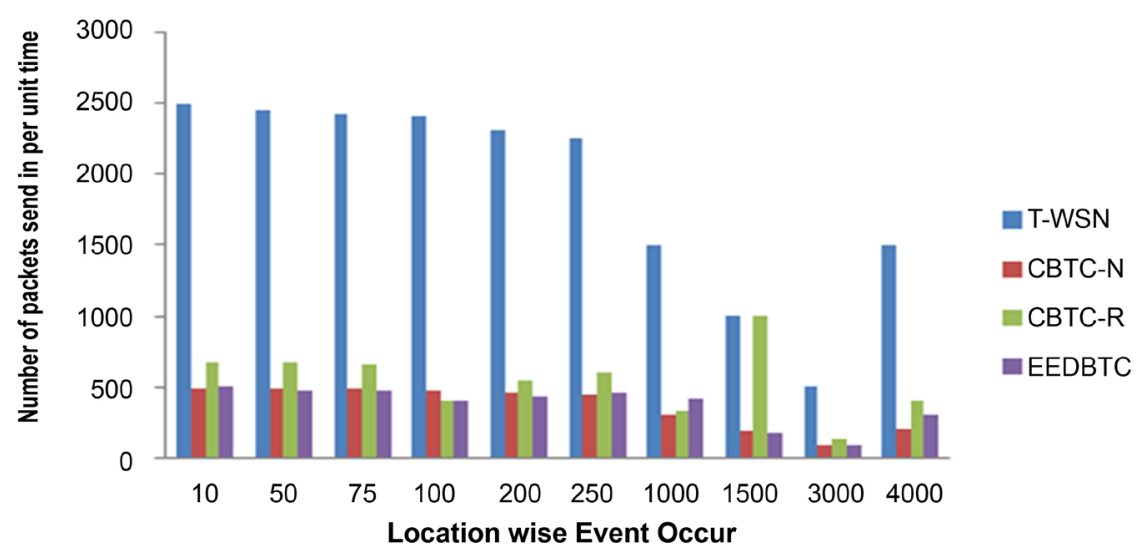

Figure 9. Comparison w.r.t. no of packets sent.

the Table 3 i.e. T-WSN is $52.46 \%$, CBTC-N $11.85 \%$, CBTC-R $14.31 \%$ and EEDBTC is $13.64 \%$. In the bellow table $10,50,75,100,200,1000,1500,3000$, 4000 are the location where event occurred and bellow the location is CPU ticks within that message passed to base station (Figure 10).

\section{Conclusion}

To minimize the consumption of energy and delay in Wireless Sensor Network, the algorithm EEDBTC shows great results. The Algorithm was evaluated using Visual Studio 12.0 in $\mathrm{C}++$. During the evaluation it was observed, that EEDBTC consume less energy as compared to other topology control algorithms i.e. T-WSN, CBTC-N and CBTC-R. Furthermore, it was observed that EEDBTC requires less 
Table 3. Evaluation of EEDBTC algorithm w.r.t. time.

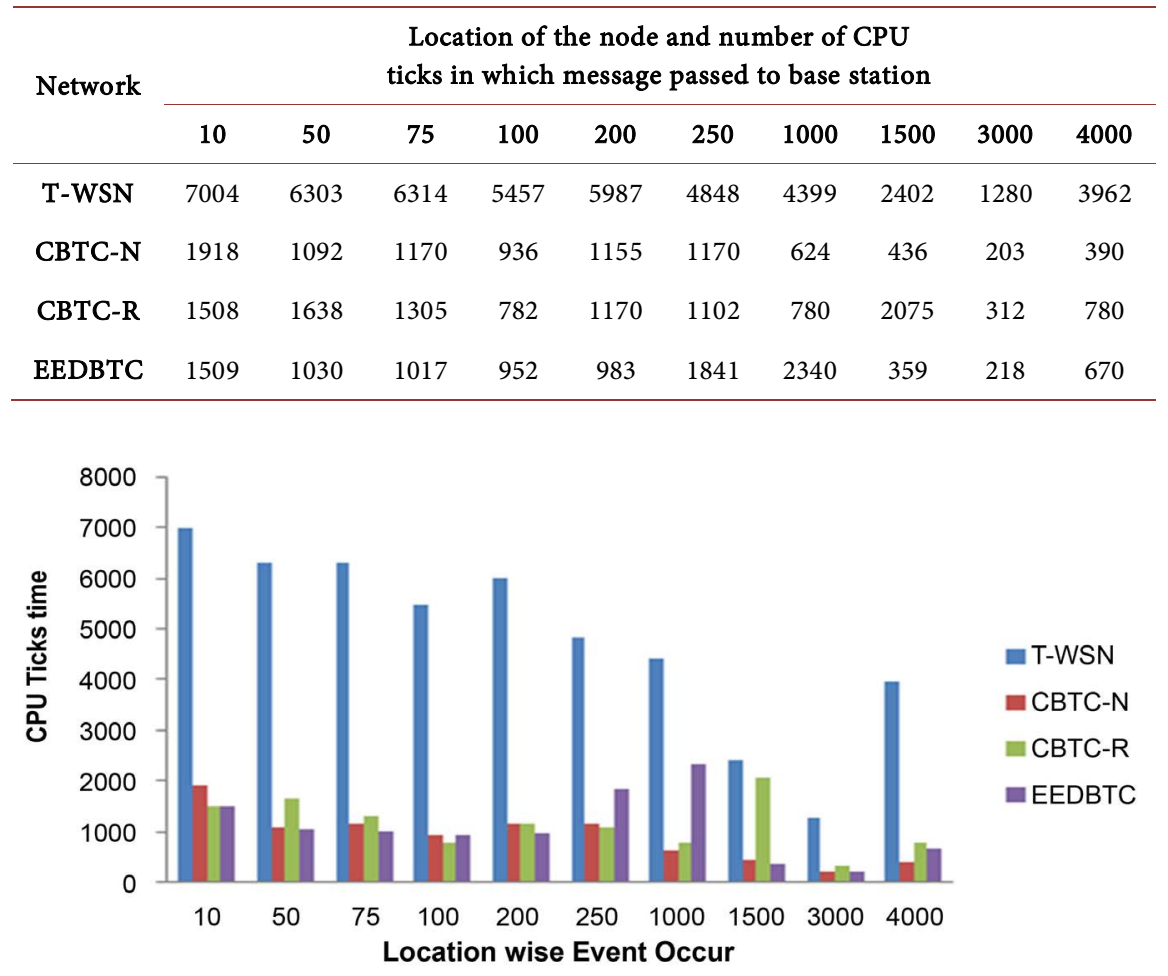

Figure 10. Comparison w.r.t. time.

CPU ticks as compared to other topology control algorithms. Similarly, EEDBTC sent minimum packets as compare to traditional wireless sensor network (TWSN), CBTC-N and CBTC-R. Using EEDBTC, we can reduce the consumption of energy of WSN and the required task can be performed in minimum amount of time.

\section{Future Work}

The proposed algorithm is based on stationary nodes. These nodes are supposed to be deployed on fixed location through normal deployment method or random deployment method. In future, the algorithm may be enhanced to conserve energy in the WSNs where nodes are capable to move from one location to other i.e. movable nodes.

\section{Conflicts of Interest}

The authors declare no conflicts of interest regarding the publication of this paper.

\section{References}

[1] Akyildiz, I.F., Su, W., Sankarasubramaniam, Y. and Cayirci, E. (2002) Wireless Sensor Networks: A Survey. Computer Networks, 38, 393-422.

https://doi.org/10.1016/S1389-1286(01)00302-4

[2] Ilyas, M. and Mahgoub, I. (2005) Handbook of Sensor Networks: Compact Wireless 
and Wired Sensing Systems. CRC Press, Boca Raton. https://doi.org/10.1201/9780203489635

[3] Raghunathan, V., Schurghers, C., Park, S. and Srivastava, M. (2002) Energy-Aware Wireless Microsensor Networks. IEEE Signal Processing Magazine, 19, 40-50. https://doi.org/10.1109/79.985679

[4] Akkaya, K. and Younis, M. (2004) Energy-Aware to Mobile Gateway in Wireless Sensor Networks. Proceedings of the IEEE Globecom, Dallas, 29 November-3 December 2004, 16-21.

[5] Anastasi, G., Coti, M., Frrancesco, M. and Passarella, A. (2009) Energy Conservation in Wireless Sensor Networks: A Survey. Ad Hoc Networks, 7, 537-568.

https://doi.org/10.1016/j.adhoc.2008.06.003

[6] Akyildiz, I.F. and Kasimoglu, I.H. (2004) Wireless Sensor and Actor Networks: Research Challenges. Ad Hoc Networks Journal, 2, 351-367. https://doi.org/10.1016/j.adhoc.2004.04.003

[7] Khan, M.A. (2013) An Energy Efficient Color Based Topology Control Algorithm for Wireless Sensor Networks. http://www.scirp.org/journal/wsn

[8] Meyer Heide, F., Schindelhauer, C., Volbert, K. and Grunewald, M. (2002) Energy, Congestion and Dilation in Radio Networks. 14th ACM Symposium Parallel Algorithms and Architectures, Winnipeg, August 2002.

[9] Santi, P. (2005) Topology Control in Wireless Ad Hoc and Sensor Networks. Wiley, New York. https://doi.org/10.1002/0470094559

[10] Rickenbach, P.V., Wattenhofer, R. and Zollinger, A. (2009) Algorithmic Models of Interference in Wireless Ad Hoc and Sensor Networks. IEEE/ ACM Transactions on Networking, 17, 172-185. https://doi.org/10.1109/TNET.2008.926506

[11] Li, X.-Y., Wang, Y., Wan, P.-J. and Frieder, O. (2004) Localized Low Weight Graph and Its Application in Wireless Ad Hoc Networks. IEEE INFOCOM, Vol. 1, 442. https://doi.org/10.1109/INFCOM.2004.1354515

[12] Li, X.-Y., Wang, Y. and Song, W.-Z. (2004) Application of k-Local MST for Topology Control and Broadcasting in Wireless Ad Hoc Networks. IEEE Transactions on Parallel and Distributed Systems, 15, 1057-1069. https://doi.org/10.1109/TPDS.2004.77

[13] Moscibroda, T. and Wattenhofer, R. (2005) Minimizing Interference in Ad Hoc and Sensor Networks. Proceedings of the 2005 Joint Work Shop on Foundations of Mobile Computing, September 2005, 24-33. https://doi.org/10.1145/1080810.1080816

[14] He, Y. and Zeng, Y. (2006) Topology Control in Wireless Sensor Networks with Interference Consideration. In: Intelligent Control and Automation, Lecture Notes in Control and Information Sciences, Vol. 344, Springer, Berlin, 202-206. https://doi.org/10.1007/11816492_28

[15] Li, L., Halpern, J.Y., Bahl, P., Wang, Y.-M. and Wattenhofer, R. (2001) Analysis of Conebased Distributed Topology Control Algorithms for Wireless Multi-Hop Networks. ACM Symposium on Principle of Distributed Computing, August 2001, 264273. https://doi.org/10.1145/383962.384043

[16] Burkhart, M., Rickenbach, P.V., Wattenhofer, R. and Zollinger, A. (2004) Does Topology Control Reduce Interference. Proceedings of ACM MOBIHOC, May 2004, 9-19. https://doi.org/10.1145/989459.989462

[17] Roy, A. and Sarma, N. (2010) Energy Saving in MAC Layer of Wireless Sensor Networks: A Survey. National Workshop in Design and Analysis of Algorithm.

[18] Younis, M. and Akkaya, K. (2008) Strategies and Techniques for Node Placement in 
Wireless Sensor Networks: A Survey. Ad Hoc Networks, 6, 621-655. https://doi.org/10.1016/j.adhoc.2007.05.003

[19] Karl, H. and Willig, A. (2005) Protocols and Architectures for Wireless Sensor Networks. John Wiley \& Sons, Hoboken. https://doi.org/10.1002/0470095121

[20] Santi, P. (2005) Topology Control in Wireless Ad Hoc and Sensor Networks. John Wiley \& Sons, Hoboken, 27-95. https://doi.org/10.1002/0470094559.ch3

[21] Suh, C., Ko, Y.-B., Lee, C.-H. and Kim, H.-J. (2006) Numerical Analysis of the Idle Listening Problem in IEEE 802.15.4 Beacon-Enable Mode. First International Conference on Communications and Networking in China, Beijing, 25-27 October 2006.

[22] Aziz, N.A.A., Aziz, K.A.B. and Ismail, W.Z.W. (2009) Coverage Strategies for Wireless Sensor Networks. World Academy of Science, Engineering and Technology, 50, 145-150.

[23] Wu, C.-H. and Chung, Y.-C. (2007) Heterogeneous Wireless Sensor Network Deployment and Topology Control Based on Irregular Sensor Model. International Conference on Grid and Pervasive Computing, Paris, 2-4 May 2007, 78-88. 